\title{
Remedial dosing regimens for delayed or missed rivaroxaban doses in patients with non-valvular atrial fibrillation based on Monte Carlo simulation
}

\author{
Xiaoqin Liu ${ }^{1}$, Yiwei Yin ${ }^{1}$, chenyu wang ${ }^{1}$, Ziran $\mathrm{Li}^{1}$, Xiao Zhu ${ }^{2}$, and Zheng Jiao ${ }^{1}$ \\ ${ }^{1}$ Shanghai Jiao Tong University Affiliated Chest Hospital \\ ${ }^{2}$ University of Otago
}

October 22, 2020

\begin{abstract}
Background: Rivaroxaban is an oral anticoagulant used widely for stroke prevention in patients with non-valvular atrial fibrillation (NVAF). During long-term anticoagulant therapy, delayed or missed doses are common. However, a lack of practical instructions on remedial methods has created a barrier to maximise the benefit of the medications. This study aimed to explore appropriate remedial dosing regimens for non-adherent rivaroxaban-treated patients. Methods: Monte Carlo simulation based on a previously established rivaroxaban population pharmacokinetic/pharmacodynamic (PK/PD) model for patients with NVAF was employed to design remedial dosing regimens. The proposed regimens were compared with remedial strategies in the European Heart Rhythm Association (EHRA) guide by assessing deviation time in terms of drug concentration, factor Xa activity, and prothrombin time under various scenarios of non-adherence. Results: The proposed remedial dosing regimens were dependent on delay duration. The missed dose should be taken immediately when the delay does not exceed 6 h; a half dose is advisable when the delay is between 6-20 h. A missed dose should be skipped if less than $4 \mathrm{~h}$ remains before the next dose. Age or renal function does not significantly influence remedial dosing regimens. The proposed regimens resulted in shorter deviation time than that of the EHRA guide in most non-adherence scenarios. Conclusion: EHRA guide may not provide optimal remedial strategies for rivaroxaban-treated non-adherent patients based on simulation. PK/PD and simulation provide valid evidence on the remedial dosing regimen of rivaroxaban for patients with NVAF, which could help to minimise the risk of bleeding and thromboembolism.
\end{abstract}

\section{Hosted file}

main text-1020.pdf available at https://authorea.com/users/369464/articles/488298-remedialdosing-regimens-for-delayed-or-missed-rivaroxaban-doses-in-patients-with-non-valvularatrial-fibrillation-based-on-monte-carlo-simulation

\section{Hosted file}

table 1014.pdf available at https://authorea.com/users/369464/articles/488298-remedialdosing-regimens-for-delayed-or-missed-rivaroxaban-doses-in-patients-with-non-valvularatrial-fibrillation-based-on-monte-carlo-simulation 


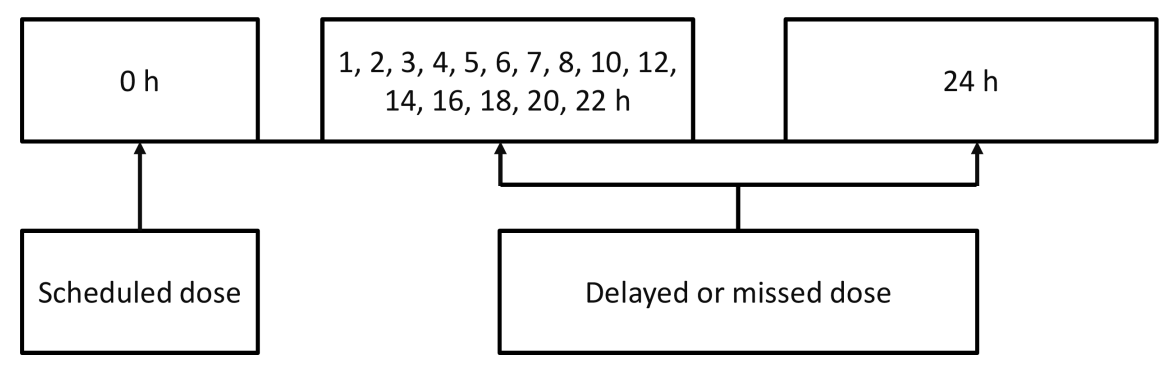

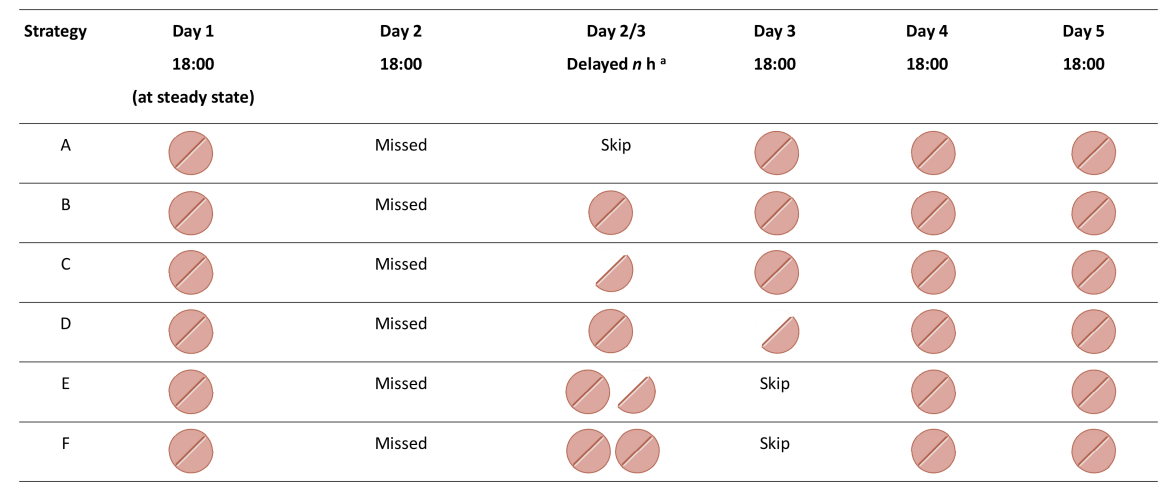

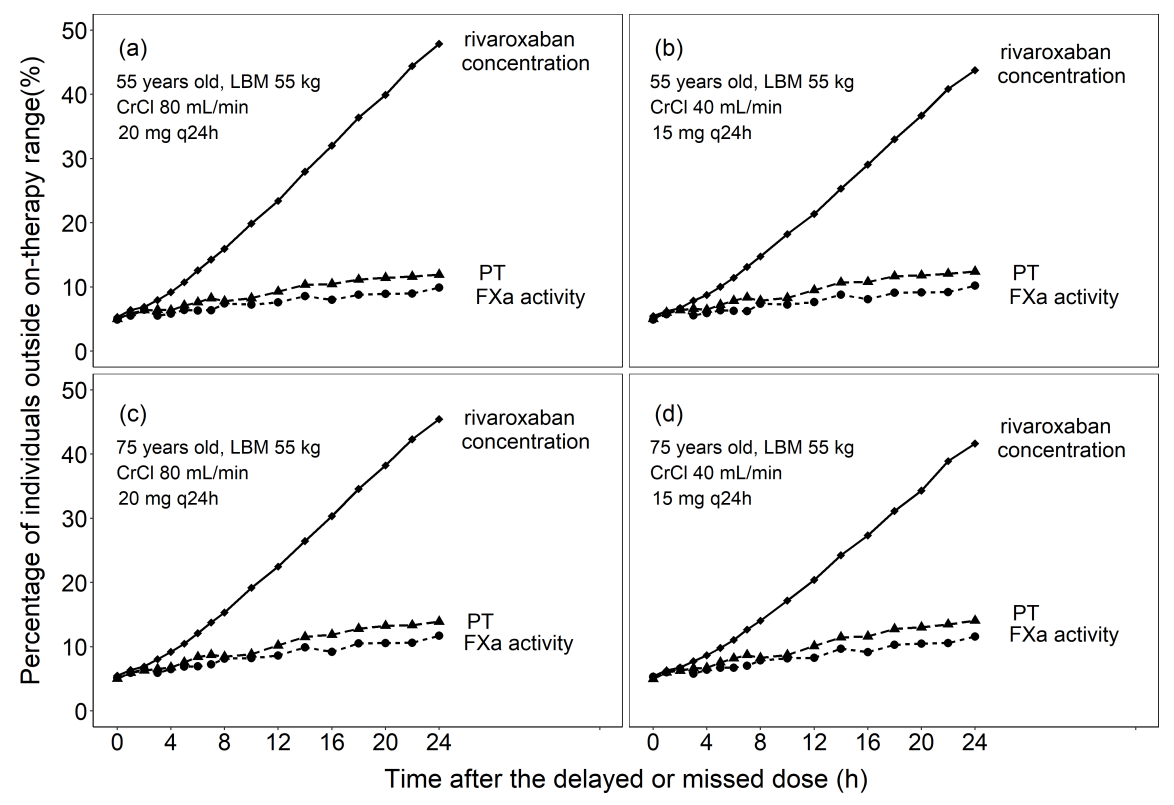



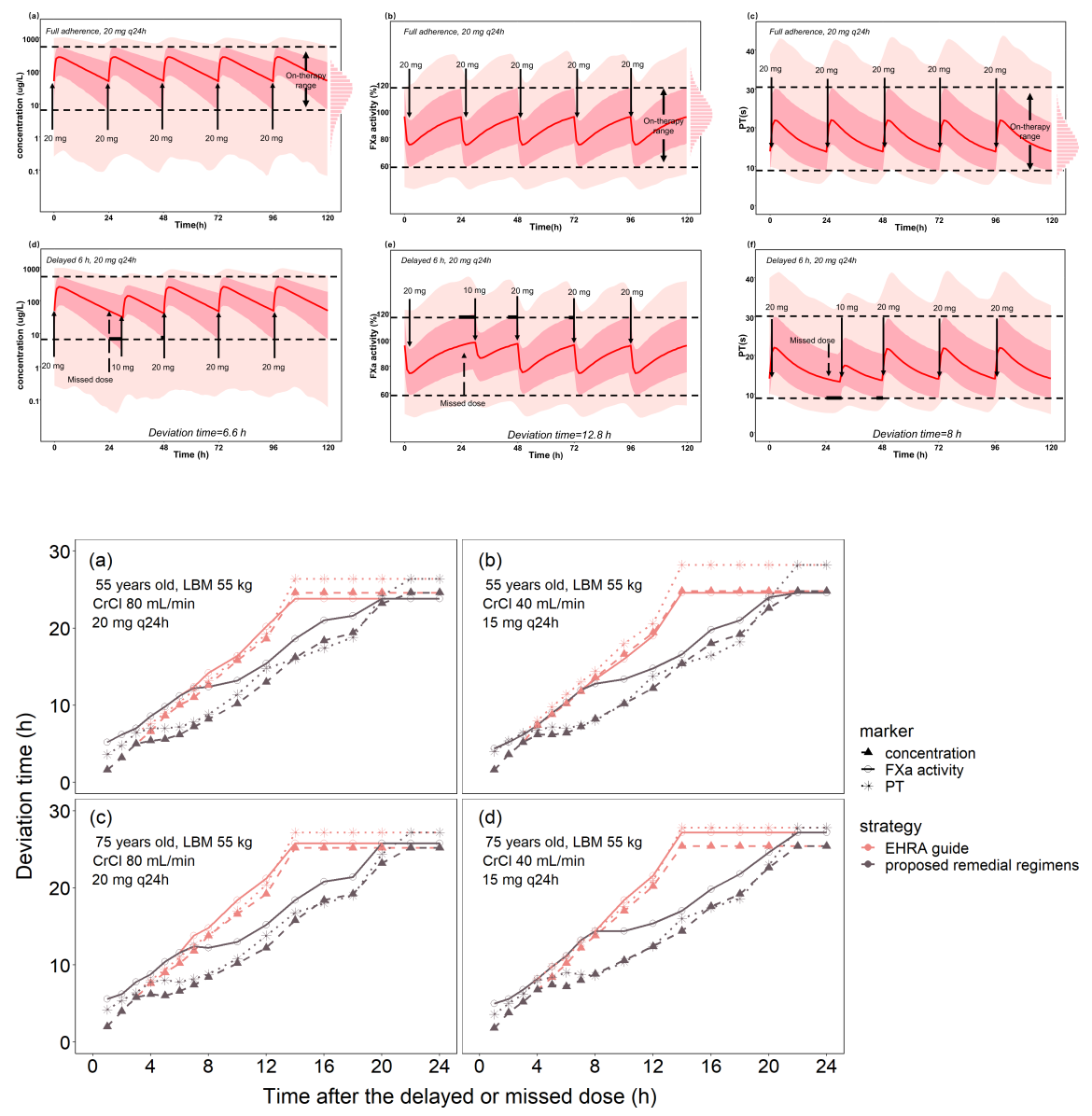